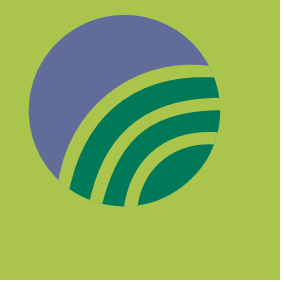

\section{Markus J. Buehler appointed MRS Bulletin Impact Editor}

\section{mrs.org/bulletin}

$\mathrm{T}$ he MRS Bulletin is pleased to announce the appointment of Markus J. Buehler, Jerry McAfee Professor in Engineering at the Massachusetts Institute of Technology (MIT), as editor of the new MRS Bulletin Impact section. In this new position, Buehler will partner with $M R S$ Bulletin Editor Gopal R. Rao and the Bulletin team to launch and develop an important new section of the journal focused on publishing high-impact original research articles, complemented by review articles and a strong set of editorial content.

"I am excited to support the materials research community in this role, to help disseminate the most innovative work that will capture the minds of researchers around the world," said Buehler. "We are presented with an extraordinary opportunity to synergistically grow $M R S$ Bulletin by converging what is available - access to a direct pipeline of the most current, advanced research through our members and meeting attendees-with what is needed-a logical source to find the most current leading research, hot topics, and foundational contributions in materials science."

The new Impact section will cover all areas of materials research and all material classes. Particular areas of interest include fundamental contributions in frontiers in materials modeling (e.g., multiscale methods, data-driven modeling, including artificial intelligence and machine learning, as well as validation and integration with experiment, digital twins), biomateriomics (e.g., living materials and materials that live, interfaces of materials and organs, biologically inspired materials, and materials for health and medicine), global impact for society, innovation and sustainability (e.g., materials for sustainability, energy, systems and integration), materials for computing and materials that compute (e.g., next-generation electronic materials and systems, smart and tunable materials), nano frontier (e.g., atom-by-atom materials synthesis and characterization, scaling and scale-up: making nano big), as well as advanced manufacturing (e.g., additive methods, self-assembly/selforganization, and autonomous materials).

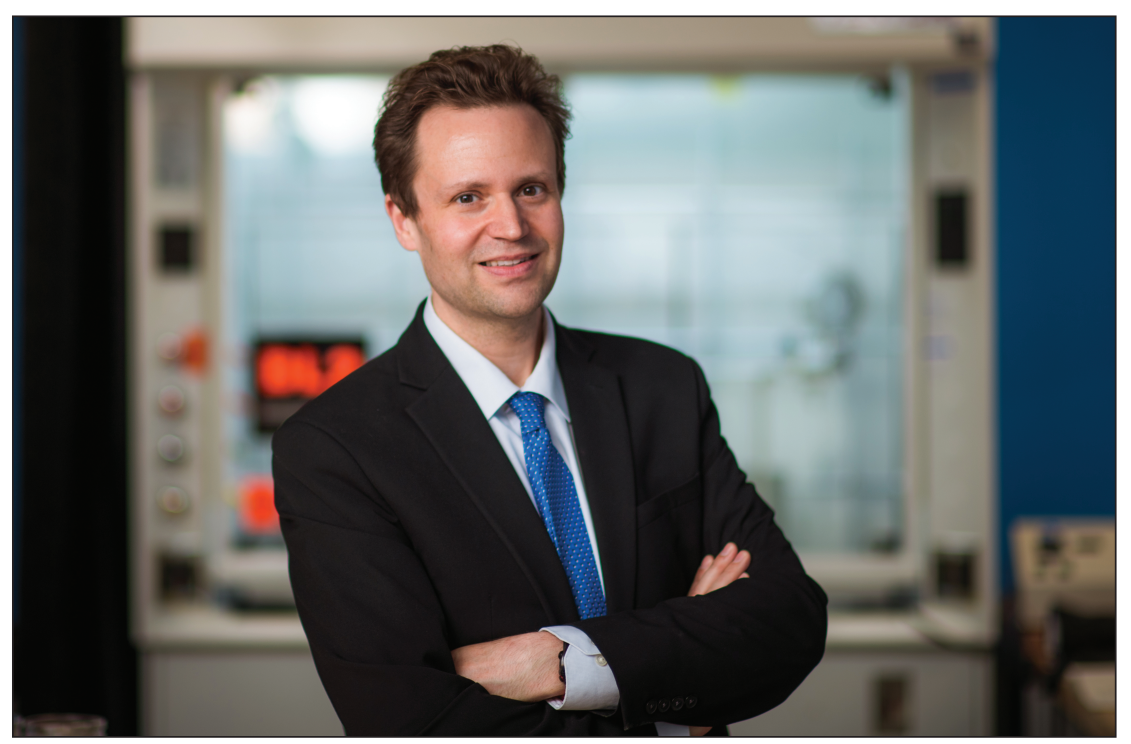

We are excited to see what frontier areas our contributors will identify, said Buehler. The Impact section will also focus on active fields in which fundamental contributions are made that are expected to shape materials applications for decades to come. We are excited to work with the community in offering new thought leadership opportunities in a variety of fields and disseminating the most important work to the world.

In his research, Buehler pursues new modeling, design, and manufacturing approaches for advanced materials that offer greater resilience and a wide range of controllable properties from the nanoto the macroscale. He enjoys a large international network of colleagues and collaborators and will utilize the journal's, as well as his own, strong connections to the materials community to identify and commission leading research and hot topics focusing on MRS meetings as a key source of content.

Buehler has a long and active history with the Materials Research Society, beginning with his graduate student days. According to Todd M. Osman, MRS Executive Director, "Markus' professional stature and extensive network are ideal for reaching into the community for cutting-edge individual research. And as a former MRS Graduate Student Award winner, the 2012 MRS Outstanding Young Investigator Award winner, and a 2021 MRS Fall Meeting chair, Buehler will also be able to advance new opportunities to connect MRS meetings and our members to MRS Bulletin."

"We are thrilled to have Markus join our MRS Bulletin team," said Rao. "The success of his candidacy is underscored by his vision for the new Impact section of the journal. He brings a breadth of expertise and an international network that will ensure its success. We look forward to working with him on this exciting new venture." 